\title{
Neuropsychiatric Features of Celiac Disease
}

\author{
Jacqueline M. Arnone ${ }^{1, *}$, Richard P. Conti ${ }^{2}$ \\ ${ }^{1}$ Department of Nursing, Kean University, Union, NJ, USA \\ ${ }^{2}$ School of Psychology, Kean University, Union, NJ, USA \\ *Corresponding author: arnonej@kean.edu
}

Received July 28, 2015; Revised August 11, 2015; Accepted August 14, 2015

\begin{abstract}
Celiac disease (CD) is a T-cell mediated genetically inherited autoimmune disease that has evolved over time. Formerly considered a pediatric disease, the rate of occurrence is now observed in individuals ranging from early childhood to senescence. Strict adherence to a gluten-free diet is the only currently available treatment for CD in order for individuals to avert future complications and malignancies. In the United States, the disease has shown widespread incidence and prevalence rates and affects $1 \%$ of the total population in not-at-risk groups. This number translates into 1 out of 133 people in the United States affected, making the disease almost 100 times more common than previously considered. While histological, genetic, incidence and prevalence of disease and quality of life research studies are replete in the extant literature, up until recently, neurological and psychological manifestations have also been studied among this cohort in relation to CD. Neurological conditions such as ataxia and neuropathy in $\mathrm{CD}$ have received the most research attention while much less attention has been paid to psychiatric conditions and cognitive functioning. The findings on cognitive functioning in CD have revolved largely around anecdotal reports and small clinical trials totaling approximately 200 patients of varying ages. Studies reporting neuropsychological test data are few and often do not include comprehensive test batteries in which to draw further conclusions on specific cognitive deficits. Larger, well-controlled studies are needed in order to form optimal diagnosis and implementation of specific support measures for individuals living with CD across the lifespan.
\end{abstract}

Keywords: celiac disease, gluten sensitivity, neuropsychiatry

Cite This Article: Jacqueline M. Arnone, and Richard P. Conti, "Neuropsychiatric Features of Celiac Disease.” International Journal of Celiac Disease, vol. 3, no. 3 (2015): 77-83. doi: 10.12691/ijcd-3-3-7.

\section{Introduction}

Celiac disease (CD) is a T-cell mediated genetically inherited autoimmune disease that has evolved over time [1]. Previously distinguished by physicians solely as a pediatric disease, the rate of occurrence is now observed in individuals ranging from early childhood to senescence [2]. Strict adherence to a gluten-free diet is the only currently available treatment for $\mathrm{CD}$ in order for individuals to avert future complications and malignancies [3]. In the United States, the disease has shown widespread incidence and prevalence rates and affects $1 \%$ of the total population in not-at-risk groups [4]. This number translates into 1 out of 133 people in the United States affected, making the disease almost 100 times more common than previously considered [1]. Additionally, the ratio of those affected increases to 1:56 of those displaying symptoms, 1:39 of individuals with second degree relatives diagnosed with $C D$, and 1:22 individuals with first-degree relatives with CD [4]. European ancestry from the Netherlands, Ireland, and Italy has been demonstrated to elevate the rate of incidence, and the disease affects females two times more frequently than males [5]. Fasano [1] reports, "work by other researchers has confirmed similar levels in many countries, with no continent spared” (p. 55). CD prevalence rates have also been reported as many as one in 86 men and 52 women based on new serology and genetic testing [6]. CD also appears to be more severe and develops more rapidly in women [7]. While histological, genetic, incidence and prevalence of disease and quality of life research studies are replete in the extant literature, up until recently, neurological and psychological manifestations have also been studied among this cohort in relation to CD.

\subsection{History}

Prior to the agricultural evolution, the nourishment of man consisted primarily of the ingestion of nuts, fruits and when available, some meat. Hunting and gathering were the principal means of survival. As the digestive tract of humans evolved over time into a more complex system that was able to accept varying and diverse foods, the way man went about getting food to eat also evolved [1]. The naissance of agrarian modernization in the Neolithic Era in how man grew and cultivated grains (primarily wheat and barley), became the nexus for the introduction of food antigens that humans had not previously been exposed to before [8]. Gastrointestinal acclimatization to these new antigens was not problematic for most, but for others, the antigens had a negative effect, giving rise to the birth of CD [1].

The naming of $\mathrm{CD}$ is accredited to the first century $\mathrm{AD}$ Greek physician Aretaeus of Cappadocia, whose writings 
spoke about "The Coeliac Affection" [9]. Naming the affection "koilaikos" from the Greek word "koelia", meaning abdomen, Aretaeus described the disease process as the inability of the stomach to keep food in it, rather it was undigested, crude and unable to be absorbed into the person's body for nutrients. The passage of this type of crude waste material deemed the individual afflicted as having coeliac [8].

In 1887, Samuel Gee, a British physician who is accredited with the origin of the contemporary history of $\mathrm{CD}$, gave a lecture on "the coeliac affection". In the lecture, Gee addressed the clinical presentation of the onset of the disease, including abdominal distention, muscular asthenia and the appearance of the individual's excrement [10]. Gee further defined the disease as "a kind of chronic indigestion which is met with in all persons of all ages, yet it is especially apt to affect children between one and five years old" [1]. Diet was believed to be both the cause and the cure for the illness, yet what specifically initiated the disease remained elusive [8].

Haas [11] published research on a study he conducted with eight children diagnosed with CD. The treatment in the experiment consisted of a banana diet. Haas's results reported the participants in the study as being clinically cured from CD, giving him enormous success among his peers, for the breakthrough in the treatment of children with CD [8]. While not explicitly stated, the diet invariably omitted gluten-containing grains, yet Haas remained adamant that all carbohydrates were the malefactors. It was not until over 40 years later that his stance was disproved [8]. A Dutch pediatrician, Dr. Wim Dicke witnessed symptom improvement in children with CD in the Netherlands during the bread shortage resulting from World War II [10]. Conversely, when bread was air dropped to the Netherlands by Allied aircraft, he witnessed a quick deterioration in the condition of the same children after they ingested bread [8]. Several years later, his work became the seminal research that documented the role gluten protein from wheat and rye food products played in causing and ultimately treating CD [10].

\section{Clinical Features}

\subsection{Signs and Symptoms}

CD can present clinically with a varying array of symptoms [12]. Ingestion of gluten can cause the individual to experience classic intestinal symptoms such as diarrhea, constipation, abdominal pain, unexplained weight loss or gain, and bloating and fatigue [6]. Additionally, individuals may also experience extraintestinal symptoms including: reproductive symptoms, dental symptoms, orthopedic symptoms and changes in blood chemistry values [12,13]. Neurological and psychological symptoms such as behavior changes, depression, anxiety, irritability, migraines and cognitive impairment have also been demonstrated in the research $[6,14,15,16]$.

Correct diagnosis is challenging as the markers of the disease ape other gastrointestinal disorders such as lactose intolerance and irritable bowel syndrome [13]. It takes an average of 10-12 years for adults to be correctly diagnosed from the initial onset of symptoms [17]. This is attributed in part to the elusiveness of the disease and its emulative characteristics of other chronic conditions.

\subsection{Disease Type}

In the late 1980s, an English epidemiologist named Dr. Logan, created a visual parallel between an iceberg and CD [18]. This pictorial analogy has been utilized in the literature to convey the intricacy of the varying degrees of the celiac state. The visible tip of the iceberg that exists above the water line depicts those individuals with either or both gastrointestinal or extra-intestinal symptoms [19]. This cadre of individuals share the representative damage of the mucosal lining of the small bowel, elevated blood antibodies against tissue transglutaminase (tTG) and at least one genetic marker for human leukocyte antigen (HLA) (HLA-DQ-2 or DQ8) required to fulfill the standards for a definitive diagnoses of the disease [18].

The next level, which lies under the sea crest illustrates individuals who like their counterparts above, demonstrate genetic predisposition, mucosal atrophy and elevated serum tTg. The difference between the two groups is that this group exhibits little or no symptoms [18]. This group is referred to as those with silent CD. The cause for this group's invisibility remains unknown [18]. While asymptomatic, this group also requires omission of gluten from their diet to prevent future symptoms, complications and malignancies [18].

Finally, the lowest of the levels represents the greatest faction of persons with a celiac form. This group also shares having the genetic marker and elevated levels of tTg with the former two groups. The difference with this group lies in the fact they have no intestinal damage, and are asymptomatic. This group is termed as having "latent" or the "potential" for developing CD [18].

\subsection{Criteria for CD}

Several criteria are principal to the pathogenesis of the disease. They are environment, genetic predisposition and immunological markers [9]. First, ingestion of any amount of gluten can cause damage to the intestinal mucosa. Therefore life-long cessation of gluten from the diet is essential to preclude future complications and malignancies.

Second, an individual must be genetically predisposed in order to have CD. Predisposition and strong correlation has been demonstrated between genes that encode inherited human leukocyte antigen (HLA), specifically the HLA-DQ2 and HLA-DQ8 alleles [9]. Fasano [20] asserts that $95 \%$ of clients with the disease demonstrate having the HLA-DQ2 allele and the remainder express positive serology for HLA-DQ8. One-third of the overall population has these alleles and the expression of either allele is required but may not be adequate enough for the development of CD [20].

HLA acts as an immunological sentinel, ascertaining other cells as either "self or non-self" [9]. Both HLA alleles categorize gluten as an alien interloper, and after gluten is identified, the alleles begin to manufacture antibodies, specifically, immunoglobulins (Ig) IgA and IgG, that ultimately damage the cells of the small bowel [9]. CD can lay in dormancy for most individuals and the disease can be activated by a variety of somatic and 
psychological stressors. [5]. Examples include viral infections, pregnancy, surgery, or even severe emotional stress.

\subsection{Diagnostic Testing}

Although routine screening is performed in Europe, the Netherlands, and Canada, routine screening is not presently performed in the United States. However, support of screening for those individuals at high risk has been demonstrated in the research literature [21]. Those at high risk include: individuals with either first or seconddegree relatives with CD, deficient IgA levels, as well as individuals who have been diagnosed with other autoimmune diseases [22].

Current serologic tests for CD include testing of antigliadin, tissue transglutaminase (tTg), deaminated gliadin peptide and antiendomysium antibodies [23]. Endoscopic biopsy of the proximal small bowel in combination with serological testing remains the benchmark for diagnosis [24,25]. Genetic testing is also available to identify the existence of the HLA genes, an important marker of whether an individual will develop CD or not [6].

\subsection{Treatment}

$\mathrm{CD}$ is a chronic condition. The only treatment to date is strict adherence to a gluten-free diet [3]. While consumption of all gluten containing foods should be avoided, fresh produce, meats, poultry and fish are allowed to be consumed as a part of this dietary regimen. There is a higher mortality rate associated with CD as a result of the increased risk of malignancies, so adherence to a gluten-free diet has been established to be the most effective treatment in reducing the risk of cancer in these individuals [26].

\section{Neuropsychiatric Implications of CD}

While classic symptom markers of CD are replete in the research literature, until recently, neurological and psychiatric manifestations have begun to be studied among this population. Current research has suggested that gluten harms the nervous system [27]. Ford hypothesized that "Gluten causes symptoms, in both CD and non-celiac gluten-sensitivity, by its action on the nervous system. This is called the gluten syndrome” (p. 438). Neurologic conditions such as ataxia, neuropathy, epilepsy, headache $[25,28,29,30]$ and psychiatric conditions such as schizophrenia, depression, anxiety, and impaired cognitive function have been the most frequently examined in children and adults with CD [31,32].

\subsection{Ataxia}

Cerebellar ataxia, with or without associated enteropathy [33], was one of the first symptoms to be described in connection with CD [34], and is one of the most frequently recognized neurological disturbances in CD $[35,36]$. Symptoms are similar to other forms of cerebellar ataxia including gait and limb ataxia [32,37,38]. Myoclonus has also been reported in patients with CD and ataxia [42].
Gluten ataxia (sporadic ataxia with positive antigliadin antibodies) was first described by Hadjivassiliou et al. [36]. Clinical features include ocular signs of cerebellar dysfunction such as gaze evoked nystagmus in over $80 \%$ of cases, upper or lower limb ataxia, gait ataxia, and dysarthria [37,40,41]. MRI findings indicate atrophy of the cerebellum and white matter hyperintensities [40]. Hadjivassiliou et al. [42] reported finding prevalent deposits of anti-tissue transglutaminase IgA antibodies in the brain, most notably in the medulla, pons and cerebellum. His findings reinforce the claim that gluten ataxia is immune mediated and therefore fits within the gluten sensitivity spectrum. Ataxic symptom relief has been reported with the adherence to a gluten-free diet $[42,43]$. Gluten ataxia is the single most common cause of sporadic idiopathic ataxia [40] and should be considered in the differential diagnosis of all patients with idiopathic sporadic ataxia [41].

\subsection{Neuropathy}

Peripheral neuropathy is the second most frequent neurologic complication in CD patients [44] and has been reported in up to $50 \%$ of cases $[28,45]$. Cooke and Smith [34] reported that among 16 CD patients, neuropathy was the main cause of death in 4 patients and a significant contributing factor in another 4 . Neuropathy may precede a diagnosis of CD $[46,47]$. Sensory axonal and small fiber sensory polyneuropathy are the most frequently reported peripheral neuropathy subtypes [48]. Research findings regarding the effect of a gluten-free diet on peripheral neuropathy have been mixed $[28,29,49]$; with some reports indicating positive outcomes $[50,51,52]$ and others reporting no significant changes [53,54].

\subsection{Epilepsy}

Several studies have demonstrated a relationship between epilepsy and CD $[55,56]$ with prevalence rates ranging from $3.5 \%$ to $7.2 \%[32,57,58]$. A meta-analysis of 15 studies [59] found a 2.1-fold increased risk of epilepsy in children with CD. However, recent data are less consistent and vary below these figures due to operational definitions of epilepsy [60]. Nevertheless, most research suggests a $2 \%-3 \%$ increased prevalence of epilepsy in CD indicating a moderate risk [60]. Higher CD prevalence rates have also been reported in epilepsy patients [55,61,62].

\subsection{Headache}

Headaches have also been reported in CD patients [28]. Gabrielli et al. [63] reported that in patients with migrane CD was found in $4.4 \%$ compared to $.4 \%$ in a control group. Zelnik et al. [32] found that headache was the most common neurologic disorder (27.9\%) in a group of 111 CD patients. A meta-analysis of 15 studies [59] found a 3.2-fold increase risk of headache in children with CD. Adherence to a gluten-free diet has been shown to have favorable outcomes on headache symptoms reported in CD $[32,49,63]$.

\subsection{Schizophrenia}

The association between schizophrenia and CD has been reported since the 1950s [64]. Dohan [65,66] recorded admissions to mental hospitals in six countries 
during World War II and noted a significant drop in patients admitted with schizophrenia that was correlated with a drop in wheat consumption. Dohan et al. [67] found that randomly assigned individuals diagnosed with schizophrenia who were admitted to a locked ward were released to an open ward more rapidly when assigned to a milk- and cereal-free diet. There were no noticeable differences when gluten was secretly added to the cerealfree diet. Another study found earlier hospital discharges after a milk- and cereal-free diet [68]. Dohan et al. [69] found only two cases of schizophrenia among 65,000 adults in remote regions of Papua New Guinea, the Solomon Islands, and on Micronesia, populations that consume little or no grain (over 130 cases would have been expected in European populations). The prevalence rate reached European levels when wheat, barley beer, and rice was introduced to these remote cultures. Recent studies have also shown a reduction in symptoms following a gluten-free diet [31]. Casella et al. [89] reported an age adjusted prevalence of $23.4 \%$ of antigliadin antibodies in 1473 people with schizophrenia which was significantly higher than general population samples of $2.9 \%$. Similar results have been reported in other studies [71,72].

\subsection{Depression and Anxiety}

Depressive and anxiety disorders are frequently reported in conjunction with CD. Carta et al. [73] found a significantly higher number of CD patients met the criteria for lifetime and 6-month major depressive disorder and panic disorder compared to controls. Other studies have reported higher rates of social phobia [74], anxiety [75,76], depression [74,77,78], and dysthymia [45]. A metaanalysis on anxiety and depression in CD found that depression is more common and more severe in adults with CD compared to normal controls. However, depression in adult CD did not differ from adults with other physical illnesses and no differences in anxiety were found. Additionally, to date there is no current evidence demonstrating a greater prevalence of anxiety found in those individuals diagnosed with CD [82]. A reduction in depressive and anxiety symptoms in CD patients have been reported following a gluten-free diet [75,79,80,81].

\subsection{Impaired Cognitive Function}

Several reports in the literature have investigated the relationship between dementia and cognitive impairment and CD [16]. Recently, brain fog, milder than cognitive impairment, has been reported in CD cases [14]. Symptoms include problems in attention, concentration, short-term memory, word-finding difficulties, and confusion. This distinction reflects the current diagnostic criteria regarding cognitive impairment.

The updated version of the American Psychiatric Association's Diagnostic and Statistical Manual of Mental Disorders, Fifth Edition (DSM-5; American Psychiatric Association [APA], 2013) [83] uses the term major and mild neurocognitive disorder for what was previously termed dementia in DSM-IV-TR [84]. According to the APA [83] a person is diagnosed with major neurocognitive disorder if the following conditions are present:
A. Evidence of significant cognitive decline from a previous level of performance in one or more cognitive domains (complex attention, executive function, learning and memory, language, perceptual-motor, or social cognition) based on:

1. Concern of the individual, a knowledgeable informant, or the clinician that there has been a significant decline in cognitive function; and

2. A substantial impairment in cognitive performance, preferably documented by standardized neuropsychological testing or, in its absence, another quantified clinical assessment.

B. The cognitive deficits interfere with independence in everyday activities (i.e., at a minimum, requiring assistance with complex instrumental activities of daily living such as paying bills or managing medications).

C. The cognitive deficits do not occur exclusively in the context of a delirium.

D. The cognitive deficits are not better explained by another mental disorder (e.g., major depressive disorder, schizophrenia) (pp. 602-603).

The same diagnostic criteria are used for mild neurocognitive disorder with evidence of modest cognitive decline.

Previous criteria [84] for a diagnosis of dementia included the development of multiple cognitive deficits manifested by both

(1) memory impairment (impaired ability to learn new information or to recall previously learned information)

(2) one (or more) of the following cognitive disturbances:
(a) aphasia (language disturbance)
(b) apraxia (impaired ability to carry out motor activities despite intact motor function)
(c) agnosia (failure to recognize or identify objects despite intact sensory function)
(d) disturbance in executive functioning (i.e., planning, organizing, sequencing, abstracting) (p. 157)

Previous studies measuring dementia used the earlier diagnostic criteria [84]. Collin et al. [85] reported five cases of patients with CD who developed dementia before age 60. Four patients had intellectual deterioration that ranged from moderate to severe. In addition to intellectual deterioration, one patient had deficits in verbal and visual memory, one had a slowing of psychomotor functions, and another had severe memory deficit, constructional deficit, and apraxia. The fifth patient had deficits in digit span, visual memory, and visuomotor constructional difficulties. Brain CT revealed diffuse cerebral or cerebellar atrophy in all patients. Kinney et al. [86] reported a case study of a 57-year-old man with CD who presented with dementia. Other neurological features included progressive neuropathy and cerebellar ataxia. The disease was fatal despite intensive medical and nutritional care.

Other studies have reported a link between dementia in individuals diagnosed with $\mathrm{CD}$ after the age of 60 . A retrospective study in Israel examined seven patients diagnosed with CD after age 60 [87]. Of the seven, two female patients were initially diagnosed with dementia of the Alzheimer type due to a progressive cognitive decline. Compliance to a gluten-free diet ameliorated the cognitive decline in both patients. Frisoni et al. [88] screened 33 
Alzheimer's patients and 24 elderly controls for CD and found no significant differences between the two groups. A total four patients tested positive for $\mathrm{CD}$; two from each group.

Casella et al. [89] examined the neuropsychological profile in CD patients 65 and over $(n=18)$ with aged matched controls $(n=18)$. All CD patients were on a gluten-free diet for a minimum of 12 months. Results indicated that $\mathrm{CD}$ patients had worse cognitive performance than controls resembling vascular cognitive impairment. Deficits were noted in semantic fluency, visuo-spatial functions, attention, and executive functions. Luostarinen et al. [90] reported a series of 10 patients with CD who presented with neurological symptoms. Two patients, both female, aged 58 and 64, suffered from memory impairment for 3 years and 2 years respectively. Neuropsychological examination revealed mild memory impairment and visuoconstructive deficits in the first patient and mild memory impairment in the second. Adherence to a gluten-free diet resulted in no changes in neurological symptoms in either patient. Hu et al. [16] reported a case series of $13 \mathrm{CD}$ patients with cognitive impairment. Amnesia was the most frequently reported cognitive complaint followed by acalculia, confusion, and personality change. The median onset of cognitive impairment was 64 years. Neuropsychological test results were available for 5 of the 13 patients and revealed learning inefficiency in 2 patients, memory deficits in 1 , and global cognitive impairment in 2 patients. Three patients were initially diagnosed with Creutzfeldt-Jakob disease due to the rapid progression of subacute dementia symptoms and 4 patients were diagnosed with celiac dementia upon evaluation.

Hallert and Astrom [91] administered a neuropsychological test battery to 19 untreated CD patients in Sweden and found no signs of cognitive impairment. Patients were divided into two groups, over 50 years $(n=10)$ and under 50 years $(n=9)$. In 4 of the patients $(21 \%)$ the test pattern was in the borderline range. Burk et al. [37] reported findings of a neuropsychological test battery administered to $8 \mathrm{CD}$ patients and 9 controls. Memory impairments were noted in immediate recall, delayed recall, and word lists. Executive dysfunction was suggested based on significant differences in verbal fluency and performance on the Wisconsin Card Sorting Test. A pilot study of 11 CD patients in Australia found that cognition is impaired in untreated CD [14]. Participants in the study had a mean age of 30 and were administered neuropsychological tests at weeks 0,12 , and 52 . Tests measuring verbal fluency, attention, and motor function significantly improved over the 12 month period following adherence to a gluten-free diet. Other researchers have also reported deficits in memory and attention in CD patients. Volta et al. [92] reported attention/memory impairment in 3 out of $13 \mathrm{CD}$ patients who presented with neurological symptoms. Zelnik et al. [32] found attention deficits in 20.7\% of CD patients examined; 13 of 64 female patients and 10 of 47 male patients.

Urban-Kowalczyk et al. [93] reported a case of a 39year-old woman presenting with anxiety, depression, apathy, fatigue, and deterioration of cognitive functions. The patient had received previous outpatient treatment for depressive and anxiety disorders. The Hamilton Depression Rating Scale was administered suggesting moderate depression. CD and microcytic anemia were diagnosed and a gluten-free diet and intramuscular iron supplementation were administered. Two neuropsychological assessments were performed with the patient in a stable mental state and without anemia. The first assessment was conducted after the CD diagnosis and second three months later. The results of the first assessment found intellectual functioning to be in the normal range and an impairment in perceptual organization. Dependent and avoidant personality features were also noted. Results of the second examination noted significant improvements in attention and psychomotor speed. An improvement in depressive and anxiety symptoms were also reported; neither were severe enough to meet the diagnostic criteria for a depressive or anxiety disorder.

Pavone et al. [94] examined the cognitive aspects of CD in children ages 2.9 to 16 years. Three groups of children were compared on measures of motor and cognitive skills, language, sociability and school performance. Group A consisted of 69 children with a mean age of 7 who were on a strict gluten-free diet. Group B consisted of 51 children with a mean age of 6.4 who were not completely compliant with the gluten-free diet. Group C were 20 age matched controls. No significant differences between groups were found on measures of motor skills, language, sociability, or school performance. Significant differences were found in visual discrimination and memory ability between Groups B and C. Four patients (25\%) in Group A scored in the impaired range on these measures.

\section{Conclusions and Further Perspectives}

Over the last several decades, our understanding of the etiology and clinical features of CD has increased substantially due to the simultaneous interest of clinical researchers around the world. Neurological conditions such as ataxia and neuropathy in $\mathrm{CD}$ have received the most research attention while much less attention has been paid to psychiatric conditions and cognitive functioning in particular. The findings on cognitive functioning in $\mathrm{CD}$ have revolved largely around anecdotal reports and small clinical trials totaling approximately 200 patients of varying ages. Studies reporting neuropsychological test data are very few and often do not include comprehensive test batteries in which to draw further conclusions on specific deficits such as executive functions. Additional research of cognitive function in CD clients could provide a deeper understanding of the cognitive issues encountered in CD including specific behaviors that may influence the condition. Larger, well-controlled studies using specific age groups are needed in order to form optimal diagnosis and implementation of specific support measures for individuals living with $\mathrm{CD}$ across the life span.

\section{References}

[1] Fasano, A., "Surprises from celiac disease," Scientific American, 301(2). 54-61. Aug. 2009.

[2] Chico, G.F., and Chico, M.A, "Demystifying celiac disease," Patient Care, 39(3). 16-20. 2005.

[3] Wagner, G., Berger, G., Sinnreich, U., Grylli, V., Schober, E., Huber, W.D., and Karwautz, A., "Quality of life in adolescents with treated celiac disease: Influence of compliance and age at 
diagnosis,” Journal of Pediatric Gastroenterology and Nutrition, 47. 555-561. 2005.

[4] Fasano, A., Berti, I., Gerarduzzi, T., Not, T., Colletti, R. B., \& Drago, S., and Horvath, K., "Prevalence of celiac disease in at-risk and not-at-risk groups in the United States," Archives of Internal Medicine, 163. 286-292. 2003.

[5] Harvard Women's Health Watch. (2006). Celiac disease: When the body goes against the grain. Retrieved from www.health.harvard.edu.

[6] Anderson, R.P., Henry, M.J., Taylor, R., Duncan, E.L., Danoy, P Costa, M.J., and Pasco, J.A., "A novel serogenetic approach determines the community prevalence of celiac disease and informs improved diagnostic pathways,” BMC Med, 11. 188. 2013.

[7] Ciacci, C., Cirillo, M., Sollazzo, R. Savino, G. Sabbatini, F. and Mazzacca G, "Gender and clinical presentation in adult celiac disease,” Scand Journal Gastroenterol, 30(11). 1077-1081. 1995.

[8] Guandalini, S., “A brief history of celiac disease,” Impact, 7(3). 12. 2007.

[9] McCabe, M.A., Toughill, E.H., Parkhill, A.M., Bossett, M.S., Jevic, M.S., \& Nye, M.L., "Celiac disease: A medical puzzle," American Journal of Nursing, 112(10). 34-44. 2012

[10] Losowsky, M.S., “A history of celiac disease,” Digestive Disease, 26. 112-120. 2008

[11] Haas, S.V., "The value of the banana in the treatment of celiac disease," American Journal of Diseases of Children, 28(4). 421437. 1924

[12] Fasano, A., "Celiac disease: How to handle a clinical chameleon," New England Journal of Medicine, 348(25). 2568-2570. 2003.

[13] Edwards, M., “Coeliac disease—an update.” Practice Nurse, 31(7). 1-6. 2006.

[14] Lichtwark, I.T., Newnham, E.D., and Robinson, S.R., "Cognitive impairment in coeliac disease improves on a gluten-free diet and correlates with histological and serological indices of disease severity," Aliment Pharmacol Ther, 40(2).160-70. 2014.

[15] Addolorato, G., Mirijello, A., D’Angelo, C., Leggio, L., Ferrulli, A., Vonghia, L., and Gasbarrini, G., "Social phobia in coeliac disease,” Scand J Gastroenterol, 43. 410-415. 2008.

[16] Hu, W.T., Murray, J.A., Greenaway, M.C., Parisi, J.E., and Josephs, K.A., "Cognitive impairment and celiac disease." Archives of Neurology, 63(10). 1440-1446. 2006.

[17] Cranney A, Zarkadas M, Graham I, Butzner, J.D. Rashid, M., Warren, R., Molloy, M., Case, S., Burrows, V., and Switzer, C., "The Canadian celiac health survey," Digestive Diseases \& Sciences, 52(4). 1087-1095. 2007

[18] Guandalini, S., “Exploring the iceberg,” Impact, 8(4). 1-2. 2009.

[19] Fasano, A., "Systemic Autoimmune Disorders in Celiac Disease," Current Opinion in Gastroenterology, 22(6). 674-679. 2006.

[20] Fasano, A., "Leaky gut and autoimmune diseases," Clinical Reviews in Allergy \& Immunology, 42(1). 71-78. Feb. 2012.

[21] Fasano, A., "European and North American populations should be screened for coeliac disease," Gut, 52(2). 168-169. 2003.

[22] Nelson, D.A., "Gluten-sensitive enteropathy (celiac disease): More common than you think," American Family Physician, 66(12). 2259-2266. 2002.

[23] Anderson, R.P., "Coeliac disease: Current approach and future prospects,” Internal Medicine Journal, 38(10). 790-799. 2008.

[24] Achkar E., Carey, W.D, Petras, R., Sivak, M.V, and Revta, R., "Comparison of suction capsule and endoscopic biopsy of the small bowel mucosa,” Gastrointestinal Endoscopy, 32. 278-281. 1986.

[25] Freeman, H.J., "Neurological disorders in adult celiac disease,” Canadian Journal of Gastroenterology \& Hepatology, 22. 909-911. 2008.

[26] Presutti, R.J., Cangemi, J.R., Cassidy, H.D., and Hill, D.A., “Celiac disease,” American Family Physician ,76(12). 1795-1802. 2007.

[27] Ford, R.P.K., “The gluten syndrome: A neurological disease,” Medical Hypotheses, 73. 438-440. 2009.

[28] Bushara, K.O., "Neurologic presentation of celiac disease," Gastroenterology, 128. S92-S97. 2005

[29] Chin, R.L., Latov, N., Green, P.H., Brannagan, T.H., Alaedini, A., and Sander, H.W., "Neurologic complications of celiac disease," Journal of Clinical Neuromuscular Disease, 5. 129-137. 2004.

[30] Hernández-Lahoz C, Mauri-Capdevila G, Vega-Villar J, and Rodrigo L., "Neurological disorders associated with gluten sensitivity,” Reviews Neurology, 53. 287-300. 2011.
[31] Jackson, J.R., Eaton, W.W., Cascella, N.G., Fasano, A., \& Kelly, D.L., "Neurologic and psychiatric manifestations of celiac disease and gluten sensitivity,” Psychiatric Quarterly, 83. 91-102. 2012.

[32] Zelnik, N., Pacht, A., Obeid, R., and Lerner, A., "Range of neurologic disorders in patients with celiac disease," Pediatrics, 113(6). 1672-1676. 2004.

[33] Ghazal, F.A., Singh, S., Yaghi, S., and Keyrouz, S.G., "Gluten ataxia: An important treatable etiology of sporadic ataxia," International Journal of Neuroscience, 122. 545- 546. 2012.

[34] Cooke, W.T., and Smith, W.T., "Neurological disorders associated with adult celiac disease,” Brain, 89. 683-722. 1966

[35] Fasano, A., "Celiac disease: How to handle a clinical chameleon," New England Journal of Medicine, 348(25). 2568-2570. 2003.

[36] Hadjivassiliou, M., Grünewald, R.A., Chattopadhyay, A.K., Davies-Jones, G.A., Gibson, A., and Jarratt, J.A., "Clinical, radiological, neurophysiological, and neuropathological characteristics of gluten ataxia," Lancet, 352(9140). 1582-1585. 1998.

[37] Burk, K., Bosch, S., Muller, C.A., Melms, A., Zuhlke, C., Stern, M., and Dichgans, J., "Sporadic cerebellar ataxia associated with gluten sensitivity,” Brain, 124. 1013-1019. 2001.

[38] Green, P.H., Alaedini, A., Sander, H.W., Brannagan, T.H., Latov, N., and Chin, R.L., "Mechanisms underlying celiac disease and its neurologic manifestations,” Cellular and Molecular Life Sciences, 62(7-8). 791-799. 2005.

[39] Bhatia, K.P., Brown, P., Gregory, R., Lennox, G.G., Manji, H., Thompson, P.D., Ellison, D.W., and Marsden, C.D., "Progressive myoclonic ataxia associated with coeliac disease: The myoclonus is of cortical origin, but the pathology is in the cerebellum," Brain, 118. 1087-1093. 1995.

[40] Hadjivassiliou, M., Grünewald, R.A., and Sharrack, B., "Gluten ataxia in perspective: Epidemiology, genetic susceptibility and clinical characteristics,” Brain, 126. 685-691. 2003.

[41] Hadjivassiliou, M., Aeschlimann, P., Strigun, A., Sanders, D.S., Woodroofe, N.M., and Aeschlimann, D., "Autoantibodies in gluten ataxia recognize a novel neuronal transglutaminase," Annals Of Neurology, 64(3). 332-343. Sep. 2008.

[42] Hadjivassiliou, M., Maki, M., Sanders, D.S., Williamson, C.A., Grünewald, R.A., Woodroofe, N.M., and Korponay-Szabó, I.R., "Autoantibody targeting of brain and intestinal transglutaminase in gluten ataxia,” Neurology, 66(3). 373-377. Feb. 2006.

[43] Hadjivassiliou, M., Davies-Jones, G. A., Sanders, D.S., and Grünewald, R.A., "Dietary treatment of gluten ataxia,” Journal of Neurology, Neurosurgery, and Psychiatry, 74(9). 1221-1224. Sep. 2003.

[44] Hadjivassiliou, M., Grunewald, R. A., and Davies-Jones, G. A., "Gluten sensitivity as a neurological illness," Journal of Neurology, Neurosurgery, and Psychiatry, 72(5). 560-563. 2002.

[45] Cicarelli, G., Della Rocca, G., Amboni, M., Ciacci, C., Mazzacca, G., Filla, A., and Barone, P., "Clinical and neurological abnormalities in adult celiac disease,” Neurological Sciences, 24(5). 311-317. 2003.

[46] Chin, R.L., Sander, H.W., and Brannagan, T.H., "Celiac neuropathy," Neurology, 60. 1581-1585. 2003.

[47] Hadjivassiliou, M., Chattopadhyay, A.K., Davies - Jones, G.A.B., Gibson, A., Grünewald, R.A., and Lobo, A.J., "Neuromuscular disorder as a presenting feature of coeliac disease," Journal of Neurology, Neurosurgery, and Psychiatry, 63(6). 770-775. Dec. 1997.

[48] Chin, R.L., and Latov, N., "Peripheral neuropathy and celiac disease," Current Treatment Options in Neurology, 7(1). 43-48. 2005.

[49] Lerner, A., Makhoul, B. F., and Eliakim, R., "Neurological manifestations of celiac disease in children and adults," European Neurological Journal, 4. 15-20. 2012.

[50] Hadjivassiliou, M., Rao, D.G., Wharton, S.B., Sanders D.S. Grünewald, R.A., and Davies-Jones, A.G., "Sensory ganglionopathy due to gluten sensitivity,” Neurology, 75(11). 1003-1008. 2010

[51] Kaplan, J.G., Pack, D., Horoupian, D., DeSouza, T., Brin, M., and Schaumburg, H., "Distal axonopathy associated with chronic gluten enteropathy: A treatable disorder,” Neurology, 38. 642-645. 1988.

[52] Polizzi, A., Finocchiaro, M., Parano, E., Pavone, P., Musumeci, S. and Polizzi A., "Recurrent peripheral neuropathy in a girl with celiac disease," Journal of Neurology, Neurosurgery, \& Psychiatry, 68(1).104-105. 2000. 
[53] Luostarinen, L., Himanen, S.L., Luostarinen, M., Collin, P., and Pirttila, T., "Neuromuscular and sensory disturbances in patients with well treated coeliac disease," Journal of Neurology, Neurosurgery, \& Psychiatry, 74. 490-494. 2003.

[54] Simonati, A., Battistella, P.A., Guariso, G., Clementi, M., and Rizzuto, N., "Coeliac disease associated with peripheral neuropathy in a child: a case report," Neuropediatrics, 29. 155158. 1998.

[55] Cronin, C.C., Jackson, L.M., and Feighery, "Celiac disease and epilepsy," Quarterly Journal of Medicine, 91. 303-308. 1998.

[56] Gobbi, G., "Coeliac disease, epilepsy and cerebral calcifications," Brain Development, 27(3). 189-200. Apr. 2005.

[57] Chapman, R.W., Laidlow, J.M., Colin-Jones, D., Eade, O.E., and Smith, C.L., "Increased prevalence of epilepsy in celiac disease," British Medical Journal, 2(6132). 250-251. 1978.

[58] Holmes, G.K.T., "Non-malignant complications of coeliac disease," Acta Paediatrica Supplement, 412. 68-75. May. 1996.

[59] Lionetti, E., Francavilla, R., Pavone, P., Pavone, L., Francavilla, T., Pulvirenti, A., Giugno, R., and Ruggieri, M., "The neurology of coeliac disease in childhood: what is the evidence? A systematic review and meta-analysis,” Developmental Medicine \& Child Neurology, 52. 700-707. 2010.

[60] Ludvigsson, J.F., Zingone, F., Tomson, T., Ekbom, A., and Ciacci, C., "Increased risk of epilepsy in biopsy-verified celiac disease: A population-based cohort study,” Neurology, 78. 1401-1407. 2012.

[61] Emami, M.H., Karimi, S., Kouhestani, S., Hashemi, M., Taheri, H., "Diagnostic accuracy of IgA anti-tissue transglutaminase in patients suspected of having coeliac disease in Iran,” Journal of Gastrointestinal and Liver Diseases,17. 141-146. 2008.

[62] Pratesi, R., Gandolfi, L., Martins, R.C., Tauil, P.L., Nobrega, Y.K., and Teixeira, W.A., "Is the prevalence of celiac disease increased among epileptic patients?” Arquivos de Neuro-Psiquiatria, 61. 330-334. 2003

[63] Gabrielli, M., Cremonini, F., Fiore, G., Addolorato, G., Padalino, C., Candelli, M., De Leo, M.E., Santarelli, L., Giacovazzo, M., Gasbarrini, A., Pola, P., Gasbarrini, A., "Association between migraine and celiac disease: results from a preliminary case control and therapeutic study," American Journal of Gastroenterology, 98. 625-629. 2003.

[64] Kalaydjian, A.E., Eaton, W., Cascella, N., and Fasano, A., "The gluten connection: The association between schizophrenia and celiac disease," Acta Psychiatrica Scandinavica, 113. 82-90. 2006.

[65] Dohan, F.C., "Wartime changes in hospital admissions for schizophrenia: A comparison of admission for schizophrenia and other psychoses in six countries during World War II," Acta Psychiatrica Scandinavica, 42. 1-23. 1966.

[66] Dohan, F.C., "Wheat consumption and hospital admissions for schizophrenia during World War II. A preliminary report," American Journal of Clinical Nutrition, 18. 7-10. 1966.

[67] Dohan, F.C., Grasberger, J.C., Lowell, F.M., Johnston, H.T., and Arbegast, A.W., "Relapsed schizophrenics: More rapid improvement on a milk- and cereal-free diet," British Journal of Psychiatry, 115. 595-596. 1969.

[68] Dohan, F.C., and Grasberger, J.C., "Relapsed schizophrenics: Earlier discharge from the hospital after cereal-free, milk-free diet," American Journal of Psychiatry, 130. 685-688. 1973.

[69] Dohan, F.C., Harper, E.H., Clark, M.H., Rodriguez, R.B., and Zigas, V., "Is schizophrenia rare if grain is rare?" Biological Psychiatry, 19. 383-399. 1984.

[70] Cascella, N.G., Kryszak, D., Bhatti, B., Gregory, P., Kelly, D.L., and Mc Evoy, J.P., "Prevalence of celiac disease and gluten sensitivity in the United States clinical antipsychotic trials of intervention effectiveness study population," Schizophrenia Bulletin, 37. 94-100. 2011.

[71] Jin, S.Z., Wu, N., Xu, Q., Zhang, X., Ju, G.Z., Law, M.H., and Wei, J., "A study of circulating gliadin antibodies in schizophrenia among a Chinese population,” Schizophrenia Bulletin, 38(3). 514518. 2012.

[72] Reichelt, K.L., and Landmark, J., "Specific IgA antibody increases in schizophrenia," Biological Psychiatry, 37. 410-413. 1995.

[73] Carta, M.G., Hardoy, M.C., Boi, M.F., Mariotti, S., Carpiniello, B., and Usai, P., "Association between panic disorder, major depressive disorder and celiac disease: A possible role of thyroid autoimmunity,” Journal of Psychosomatic Research, 53. 789-793. 2002.

[74] Addolorato, G., Mirijello, A., D’Angelo, C., Leggio, L., Ferrulli, A., Vonghia, L., and Gasbarrini, G., "Social phobia in coeliac disease,” Scandinavian Journal of Gastroenterology, 43. 410-415. 2008.

[75] Addolorato, G., Capristo, E., Ghittoni, G., Valeri, C., Masciana, R and Ancona, C., "Anxiety but not depression decreases in coeliac patients after one-year gluten-free diet: A longitudinal study," Scandinavian Journal of Gastroenterology, 36. 502-506. 2001.

[76] Hauser, W., Janke, K.H., Klump, B., Gregor, M., and Hinz, A., "Anxiety and depression in adult patients with celiac disease on a gluten-free diet," World Journal of Gastroenterology, 16. 27802787. 2010.

[77] Ludvigsson, J.F., Reutfors, J., Osby, U., Ekbom, A., and Montgomery, S.M., "Coeliac disease and risk of mood disordersa general population-based cohort study," Journal of Affective Disorders, 99. 117-126. 2007.

[78] Ruuskanen, A., Kaukinen, K., Collin, P., Huhtala, H., Valve, R., and Maki, M., "Positive serum antigliadin antibodies without celiac disease in the elderly population: Does it matter?" Scandinavian Journal of Gastroenterology, 45. 1197-1202. 2010.

[79] Corvaglia, L., Catamo, R., Pepe, G., Lazzari, R., and Corvaglia, E., "Depression in adult untreated celiac subjects: Diagnosis by the pediatrician,” American Journal of Gastroenterology, 94. 839-843. 1999.

[80] Pynnönen, P.A., Isometsä, E.T., Verkasalo, M.A., Savilahti, E., and Aalberg, V.A., "Untreated celiac disease and development of mental disorders in children and adolescents," Psychosomatics, 43(4). 331-334. 2002.

[81] Pynnönen, P.A., Isometsä, E.T., Verkasalo, M.A., Kähkönen, S.A., Sipilä, I. Savilahti, E., and Aalberg, V.A., "Gluten-free diet may alleviate depressive and behavioural symptoms in adolescents with coeliac disease: a prospective follow-up case-series study," BMC Psychiatry, 5. 14. 2005.

[82] Smith, D. F., \& Gerdes, L. U., "Meta-analysis on anxiety and depression in adult celiac disease," Acta Psychiatrica Scandinavica, 125(3), 189-193. 2012. doi:10.1111/j.16000447.2011.01795.x

[83] Diagnostic and Statistical Manual of Mental Disorders, 5th Edition Washington, DC, American Psychiatric Association, 2013.

[84] Diagnostic and Statistical Manual of Mental Disorders, 4th Edition, Text Revision. Washington, DC, American Psychiatric Association, 2000.

[85] Collin, P., Pirttila, T., Nurmikko, T., Somer, H., Erila, T., and Keyrilainen, O., "Celiac disease, brain atrophy, and dementia," Neurology, 4. 372-375. 1991.

[86] Kinney, H.C., Burger, P.C., Hurwitz, B.J. Hijmans, J.C. and Grant, J.P., "Degeneration of the central nervous system associated with celiac disease,” Journal of the Neurological Sciences, 53(1). 9-22. 1982.

[87] Lurie, Y., Landau, D.A., Pfeffer, J., and Oren, R., “Celiac disease diagnosed in the elderly," Journal of Clinical Gastroenterology, 42(1). 59-61. 2008.

[88] Frisoni, G.B., Carabellese, N., Longhi, M., Geroldi, C., Bianchetti, A., Govoni, S., Cattaneo, R., and Trabucchi, M., "Is celiac disease associated with Alzheimer's disease?” Acta Neurologica Scandinavica, 95(3). 147-151. 1997.

[89] Casella, S., Zanini, B., Lanzarotto, F., Caselani, F., Magni, A., Romanelli, G., and Lanzini, A., "Neuropsychological pattern during gluten free diet (GFD) in elderly coeliac patients," Gut, 60 . A86. 2011.

[90] Luostarinen, L., Pirttilä, T., and Collin, P., "Coeliac disease presenting with neurological disorders,” European Neurology, 42(3), 132-135. 1999.

[91] Hallert, C., and Astrom, J., "Intellectual ability of adults after lifelong intestinal malabsorption due to celiac disease," Journal of Neurology, Neurosurgery, and Psychiatry, 46(1), 87-89. 1983.

[92] Volta, U., De Giorgio, R., Petrolini, N., Stangbellini, V., Barbara, G., Granito, A., DePonti, F., Corinaldesi, R, and Bianchi, F.B., "Clinical findings and anti-neuronal antibodies in coeliac disease with neurological disorders," Scandinavian Journal of Gastroenterology, 37(11). 1276-1281. Nov. 2002.

[93] Urban-Kowalczyk, M., Smigielski, and Gmitrowicz, A., "Neuropsychiatric symptoms and celiac disease," Neuropsychiatric Disease and Treatment, 10. 1961-1964. 2014.

[94] Pavone, L., Mazzone, D., Incorpora, G., Drago, F., and Bottaro, G., "Cognitive disturbances in children with coeliac disease," In G. Gobbi, F. Andermann, S. Naccarato, G. Banchini, Eds. Epilepsy and other neurological disorders in coeliac disease. London, England: Libbey. 1997, 291-293. 